\title{
Splenic abscess complicating gastroenteritis due to Salmonella Virchow in an immunocompetent host
}

\author{
"Splenic \\ abscesses \\ should be \\ suspected in \\ patients with \\ unexplained \\ fevers and \\ left upper \\ quadrant pain"
}

\section{Clinical record}

A 20-year-old man was admitted to a regional hospital with fevers, rigors, anorexia and left upper quadrant pain. It was his fourth presentation to the emergency department in the preceding 10 days. On the first two presentations, he had been sent home with a provisional diagnosis of renal colic After review by his general practitioner, he had undergone outpatient imaging that identified filling defects in the pulmonary arteries of his left lower lobe, which were reported as being consistent with pulmonary emboli. In addition, two hypodense splenic lesions were identified, as well as collapse and possible consolidation of the left lower lobe. His GP had referred him to the emergency department for further review (his third presentation), after which he had commenced therapeutic anticoagulation for a presumed diagnosis of pulmonary emboli.

The patient's history was notable for a self-limiting episode of gastroenteritis 6 weeks before his initial presentation, with sick family contacts. On his fourth presentation, he described progressive left upper quadrant and flank pain over the preceding 10 days, with intermittent fevers and rigors. He had no other focal infective symptoms on review.

On examination, he was found to have a fever (temperature, $39.3^{\circ} \mathrm{C}$ ), sinus tachycardia (heart rate, 154 beats $/ \mathrm{min}$ ), tachypnoea (respiratory rate, 28 breaths/min), hypotension (blood pressure, $97 / 66 \mathrm{mmHg}$ ), decreased breath sounds at the left base of his lung fields and mild left upper quadrant tenderness. Investigations showed a white cell count of $16 \times 10^{9} / \mathrm{L}$ (reference interval [RI], 4.0-11.0 × 10\%/L), with a predominant neutrophilia (neutrophils, $14 \times 10 \% / \mathrm{L}$ [RI, $\left.2.0-7.0 \times 10^{9} / \mathrm{L}\right]$ ). Results of his liver function tests and electrolyte, urea and creatinine levels were all within reference intervals.

A computed tomography scan of the chest and upper

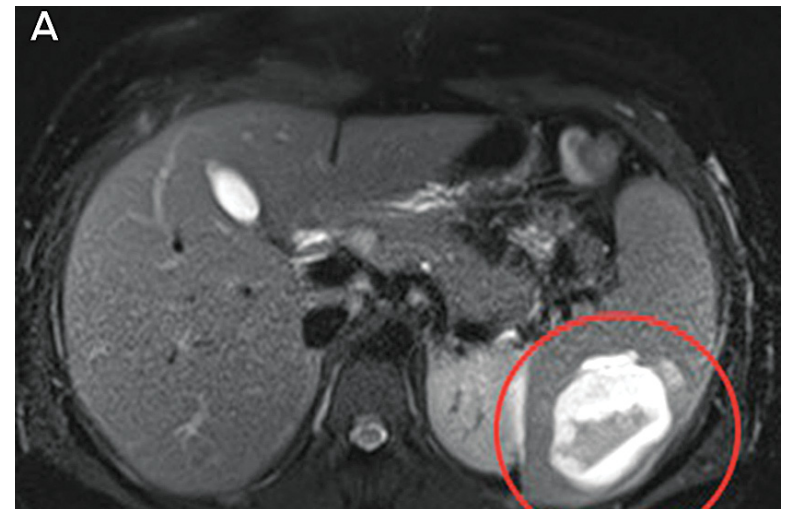

abdomen again showed two low-density lesions of unclear aetiology in the spleen, as well as a left-sided pleural effusion and collapse of the left lower lobe. Given the possibility that the hypodense splenic lesions represented septic emboli from a cardiac source, the patient was treated empirically with benzylpenicillin, flucloxacillin and gentamicin for a provisional diagnosis of endocarditis. However, a transthoracic echocardiogram performed the next day did not support this diagnosis, with no abnormalities detected. Beyond the radiological findings described, there were no other clinical grounds to support a diagnosis of endocarditis.

Blood cultures taken on Day 1 of admission were positive for gram-negative bacilli, with confirmation of a non-typhoidal Salmonella species (later confirmed as Salmonella Virchow) the following day. This allowed targeted antibiotic therapy, once susceptibilities were known, with ampicillin ( 2 g every 6 hours). Cultures of stool samples taken at admission were positive for the same isolate, consistent with the patient's self-limiting episode of gastroenteritis 6 weeks before his first presentation.

Magnetic resonance imaging of the abdomen suggested that the two splenic lesions were likely to represent abscesses in this clinical context (Figure). Given our patient's ongoing sepsis, a decision was made to perform a laparoscopic splenectomy for source control on Day 5 of admission. Surgical specimens tested positive for Salmonella Virchow. Histopathological testing identified cystic lymphangiomas of the spleen. Despite problems with postoperative pain and a prolonged ileus, the patient made a full recovery. He received appropriate post-splenectomy vaccinations, along with a total of 2 weeks' intravenous ampicillin, followed by a 2-week course of oral amoxicillin.
Jonathan R Sillar BEng(Hons)/BCom MB BS(Hons)'

Aiveen M Bannan MB BS, FRACP, MPH²

Leigh Dahlenburg BMedSc, MB BS(Hons), FRACP²

1 East Coast Medical Network Sydney, NSW.

2 Port Macquarie Base Hospital, Port Macquarie, NSW.

jonsillar@icloud.com
A: Axial T2-weighted magnetic resonance image (MRI) of the abdomen, without contrast, showing an abscess in the inferior pole of the spleen (circle). B: Saggital T2-weighted MRI of the abdomen, without contrast, showing two splenic abscesses (circles).

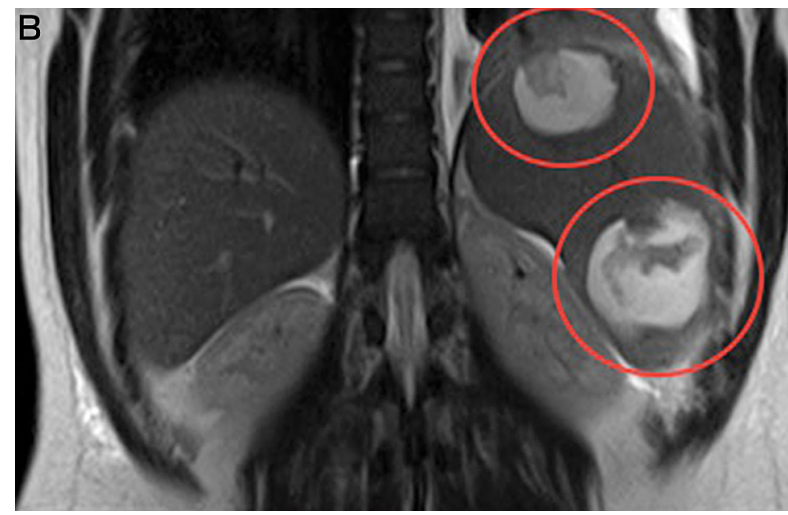

N on-typhoidal salmonellae are common foodborne pathogens. In Australia, they are the second most frequent bacterial isolates identified in cases of acute gastroenteritis, after Campylobacter jejuni. In 2010, OzFoodNet sites reported 11992 cases of Salmonella infection, a rate of 53.7 cases per $100000 .{ }^{1}$
Salmonella Virchow was the third most common isolate, after Salmonella Typhimurium and Salmonella Enteritidis. Non-typhoidal Salmonella infection outbreaks are most commonly associated with consumption of poultry and eggs, but have also been linked to fresh produce and, increasingly, contact with pet reptiles. ${ }^{2}$ 
Up to $8 \%$ of patients with gastroenteritis secondary to non-typhoidal Salmonella infection develop bacteraemia. ${ }^{3}$ Risk factors for invasive infection include extremes of age, immunosuppressed states, malignancy, HIV infection and use of tumour necrosis factor-blocking medication. ${ }^{4}$ Our case is unusual in that bacteraemia occurred in an otherwise immunocompetent host.

Extraintestinal focal infections have been reported to occur in $5 \%$ to $10 \%$ of patients with non-typhoidal Salmonella bacteraemia. ${ }^{3}$ The best recognised complications are endovascular infections, most commonly involving the aorta, that result from seeding of atherosclerotic plaques and aneurysms. ${ }^{5}$ However, focal infections of almost all organ systems have been reported.

Splenic abscesses are most commonly seen as a complication of infective endocarditis, occurring in about $5 \%$ of patients. ${ }^{6,7}$ They are also found as a rare complication of non-typhoidal Salmonella infections. In one case series of 49 patients from southern Taiwan, Salmonella species were the third most common pathogens isolated from splenic abscesses, accounting for $11 \%$ of cases. ${ }^{8}$ The most common presentations among the 49 patients with splenic abscesses were fever (47 patients), abdominal pain confined to the left upper quadrant (33 patients), left pleural effusion and splenomegaly (both 27 patients), all of which were present in our patient.

About $50 \%$ of patients presenting with splenic abscesses have pre-existing anatomical abnormalities. ${ }^{9}$ The cystic lymphangiomas identified in our patient almost certainly predisposed him to developing splenic abscesses.

According to the literature, the mainstay of treatment for splenic abscesses is splenectomy. Data from 287 cases published between 1987 and 1995 suggested that non-operative management, which included invasive treatment with percutaneous aspiration and catheter drainage, had a success rate of less than $65 \% .{ }^{10}$ The same series suggested that antimicrobial therapy alone had a success rate of less than 50\%. Salvage splenectomy, however, was not shown to result in increased mortality. Another retrospective study of 51 patients in a tertiary hospital between 1998 and 2003 reported survival rates of $48 \%$ with antimicrobial therapy alone, $45 \%$ with pigtail catheter insertion and drainage in

Lessons from practice

- Splenic abscesses are a rare but potentially lifethreatening complication of non-typhoidal Salmonella bacteraemia.

- Splenic abscesses should be considered as a possible source of infection in patients presenting with unexplained fevers and left upper quadrant or left flank pain.

- Splenectomy plus appropriate antimicrobial therapy remains the mainstay of treatment for splenic abscesses.

- Interventional radiological techniques should be considered as a spleen-preserving strategy on a caseby-case basis and where experienced radiologists are available. addition to antimicrobial therapy, and 100\% with splenectomy and antimicrobial therapy. ${ }^{11}$ These results may be influenced by selection bias but do suggest improved outcomes with splenectomy over less invasive strategies.

Splenic abscesses are a rare but serious complication of non-typhoidal Salmonella bacteraemia that may occur in otherwise immunocompetent individuals. Splenic abscesses should be suspected in patients with unexplained fevers and left upper quadrant pain. The mainstay of treatment is splenectomy with appropriate antimicrobial therapy.

\section{Competing interests: No relevant disclosures. \\ C 2015 AMPCo Pty Ltd. Produced with Elsevier B.V. All rights reserved.}

References are available online at www.mja.com.au. 
1 OzFoodNet Working Group. Monitoring the incidence and causes of diseases potentially transmitted by food in Australia: annual report of the OzFoodNet network, 2010. Commun Dis Intell Q Rep 2012; 36: E213-E241.

2 Marcus R, Varma JK, Medus C, et al. Re-assessment of risk factors for sporadic Salmonella serotype Enteritidis infections: a case-control study in five FoodNet Sites, 2002-2003. Epidemiol Infect 2007; 135: 84-92.

3 Mandal BK, Brennand J. Bacteraemia in salmonellosis: a 15 year retrospective study from a regional infectious diseases unit. BMJ 1988; 297: 1242-1243.

4 Gordon MA. Salmonella infections in immunocompromised adults. J Infect 2008; 56: 413-422.

5 Benenson S, Raveh D, Schlesinger Y, et al. The risk of vascular infection in adult patients with nontyphi Salmonella bacteremia. Am J Med 2001; 110: 60-63.

6 Robinson SL, Saxe JM, Lucas CE, et al. Splenic abscess associated with endocarditis. Surgery 1992; 112: 781-786.

7 Johnson JD, Raff MJ, Barnwell PA, Chun CH. Splenic abscess complicating infectious endocarditis. Arch Intern Med 1983; 143: 906-912.

8 Lee CH, Leu HS, Hu TH, Liu JW. Splenic abscess in southern Taiwan. J Microbiol Immunol Infect 2004; 37: 39-44.

9 Chun CH, Raff MJ, Contreras L, et al. Splenic abscess. Medicine (Baltimore) 1980; 59: 50-65.

10 Ooi LL, Leong SS. Splenic abscesses from 1987 to 1995. Am J Surg 1997; 174: 87-93.

11 Tung CC, Chen FC, Lo CJ. Splenic abscess: an easily overlooked disease? Am Surg 2006; 72: 322-325. 